\title{
Customized Arch Support Insoles Improve Physical Functional Performance in Children: A Randomized Controlled Study
}

\section{Ying-Chen Kuo}

Shin Kong Wu Ho Su Memorial Hospital

Ru-Lan Hsieh ( M001052@ms.skh.org.tw)

Shin Kong Wu Ho Su Memorial Hospital https://orcid.org/0000-0002-6228-9896

Wen-Chung Lee

National Taiwan University College of Public Health

\section{Research Article}

Keywords: Foot orthoses, child, physical functional performance, International Classification of Functioning, Disability and Health

Posted Date: October 13th, 2021

DOI: https://doi.org/10.21203/rs.3.rs-964161/v1

License: (9) This work is licensed under a Creative Commons Attribution 4.0 International License. Read Full License 


\section{Abstract}

The effectiveness of arch-support insoles on children remains controversial. We evaluated the short-term therapeutic effects of arch-support insoles on the physical functional performance, physical function, and psychological well-being of healthy children under the International Classification of Functioning, Disability, and Health (ICF) framework. It was a prospective double-blind randomized controlled study. The participants were randomized into 2 groups, the treatment group (with customized arch-support insoles) and the control group (without insoles) for a 12-week intervention period. The walking speed test, stairs ascent and descent tests, the Five Times Sit-to-Stand test, and the Timed Up and Go test for physical functional performance, and the Pediatric Outcome Data Collection Instrument-Parent (PODCl) and the Child Health Questionnaire-Parent Form (CHQ-PF28) for the physical function and psychological well-being of children were assessed. Forty-five children completed the study. Compared with the control group, after 12 weeks of wearing customized insoles, the treatment group exhibited significant improvement in physical functional performance in terms of fastest walking speed (effect size: $.515, P=$ .046 ), stairs ascent time (effect size: .658, $P=.023$ ), and stairs descent time (effect size: .718, $P=.012$ ). No significant difference was found between PODCl and CHQ-PF28 scores.

Conclusions: Children wearing customized arch-support insoles for 12 weeks improved their physical functional performance which belonged to the domain of activity in the ICF. However, using insoles did not affect the physical function and psychological well-being of children.

ClinicalTrials.gov (NCT03198299), date of registration: June 2017, retrospectively registered.

\section{What Is Known}

1. Customized arch-support insoles improved pain and physical function in children with symptomatic flexible flat feet.

2. The effect of applying foot orthotics in healthy and asymptomatic children remains controversial.

\section{What Is New}

1. Using customized arch-support insoles in health children demonstrated better physical functional performance. 2. No significant effect was found on physical function or psychological well-being in the group with insoles during 12-week follow-up.

\section{Introduction}

In children with normal growth, the medial longitudinal arch (MLA) of the foot starts growing between ages 2 and 3 and develops rapidly by the age of $6[1,2]$. In school-aged children, considerable changes continue until late adolescence [3]. Furthermore, the leg axis of children changes from varus to valgus 
between the ages of 2 and 6 , and final neutralization is reached by the age of 6 [4]. Contributing to lower limb stability, the tendons and ligaments continue to grow and strengthen until puberty [4].

Weight, gender, age, height, foot progression angle, and joint laxity are factors that increase the stress load on the foot and thus affect the foot arch $[5,6]$. Clinically, the most common foot problem in children is flexible flat foot, defined by MLA collapse during weight-bearing and rebound after unloading. The prevalence of flexible flat feet in preschool children is $44 \%$ but decreases with age [7]. Only $24 \%$ and $28 \%$, respectively, of 6-year-olds and 5- to 13-year-old children had flat feet, a finding compatible with the natural course of MLA development. In addition, the prevalence of hypermobility in children ranges from $3-30 \%$ and decreases with age [8]. Generalized joint hypermobility is observed more often in girls than boys, specifically at a $3: 1$ ratio [8].

For nonsurgical treatment, foot insoles are widely used for symptomatic flexible flat feet in children. We previously demonstrated that customized arch-support insoles improve pain and physical function in children with symptomatic flexible flat feet [9]. However, many parents choose commercially available shoes with medial arch support for asymptomatic children. One relevant report determined that insoles with added medial support may be unnecessary for children's shoes because of the natural course of MLA development [4], but another concluded that the application of foot orthotics may have a positive impact on pain, foot posture, gait, function, and structural and kinetic measures [10]. Lee et al reported improved pain and balance in 24 children with symptomatic flexible flat feet during a 3-month intervention [11]. By contrast, a randomized controlled trial reported that foot insoles with toe-grip bars improved running speed over a 4-week intervention period without affecting the sense of balance in preschool children [12].

The International Classification of Functioning, Disability, and Health (ICF) framework is a useful tool for describing human functioning and disability and has been widely applied clinically. It demonstrates the interactions between body functions and structures, activity and participation, and personal and environmental factors [13]. Because the effect of foot insoles with medial arch support on asymptomatic healthy children with or without flexible flat feet remains unclear, we hypothesized that short-term use of customized foot insoles with arch support may improve the physical functional performance and physical function in such children based on the ICF framework.

\section{Materials And Methods}

\section{Study Design and Randomization}

This was a prospective, randomized controlled clinical trial followed CONSORT guidelines and was approved by the Ethics Committee of Shin Kong Wu Ho-Su Memorial Hospital in Taiwan. The study was registered at ClinicalTrials.gov (NCT03198299). Written informed consent was obtained from the parents of participating children, who were subsequently randomly divided into 2 groups. Randomization was accomplished using computer-generated numbers with a block size of 4 . When initially recruited, each participant received a sealed envelope revealing their group allocation. The participants were then 
randomly assigned to the treatment group (with customized medial arch-support insoles) and the control group (without customized medial arch-support insoles). The control group members were told they were on a 12-week waiting list to receive the customized insoles. All participants were blinded to their group allocation. An investigator blinded to group allocation evaluated the basic demographics, physical functional performance, physical function, and psychological well-being at the baseline and 12 weeks after the intervention (Figure 1).

\section{Participants}

From January 2016 to December 2016, a total of 45 children were recruited from the outpatient clinic at the Department of Physical Medicine and Rehabilitation, Shin Kong Wu Ho-Su Memorial Hospital in Taiwan. The children were brought by their parents for a regular physical check-up or for musculoskeletal counseling and had no complaints of any lower limb pain. The inclusion criteria of this study were healthy children from ages 3 to 10 who could walk independently for 15 meters. We excluded children with a confirmed diagnosis of developmental delay or pathological flat foot, previous foot surgeries or injuries, foot structural deformity, or neurological disorder.

\section{Baseline Assessment}

The investigator and the assessor were blinded to the group allocations. The participants of both groups and their parents were also blinded to their group allocations. Baseline characteristics including age and sex were collected, and body mass index was measured.

\section{Clinical Measurements}

\section{Beighton Hypermobility Score}

To evaluate the hypermobility of joints, we used the Beighton hypermobility score, which is based on a 9point scale. The examination included 4 passive range of motion tests of the bilateral joints and 1 trunk forward-flexion test. The cut-off value for generalized hypermobility was $>4$, consistent with other research [8]. The intraobserver and interobserver reproducibility were satisfactory. ${ }^{14}$

\section{Navicular Drop Test}

The navicular drop test was used to evaluate the MLA. Navicular height was defined as the distance between the most prominent part of the navicular tuberosity and the supporting surface. The change of navicular height was observed in 2 conditions: subtalar joint in a neutral position and full weight-bearing in a relaxed state. Normal values for the navicular drop test for boys and girls are 4 to $6 \mathrm{~mm}$ and 3 to 4 $\mathrm{mm}$, respectively [17]. Pronated foot was defined as a more than 9-mm drop [17]. The test reported satisfactory intrarater and interrater reliability (.83 to .95) [17].

\section{Foot Posture Index}


We used the Foot Posture Index to determine the foot position. The child was instructed to stand with arms relaxed at their sides. The position of the joints and the deviation of the foot and ankle were observed. This test consisted of 6 items scored on a 5 -point scale from -2 to +2 . The final score was defined as highly pronated $(+10$ to +12$)$, pronated $(+6$ to +9$)$, normal $(0$ to +5$)$, supinated $(-1$ to -4$)$, and highly supinated $(-5$ to -12$)$. Satisfactory interrater reliability has previously been reported [17].

\section{Intervention}

We provided information on the natural history of the pediatric flexible flat foot to the parents and children when they were initially recruited. Each participant in the treatment group was evaluated by a qualified physiatrist who used a heat gun to prepare customized foot insoles according to the children's feet alignment. The subtalar joint was maintained in a neutral position, and the forefoot and hindfoot were not pronated or supinated during the process. The physiatrist first used a right thumb and index finger positioned at the talar head medially and laterally to maintain the subtalar joint in a neutral position [18]. Next, the physiatrist affixed the heated insole so that it adhered completely to the foot contour to enhance the MLA of the foot. During the process, the participant remained in a standing position to ensure the insole stayed in full contact with the sole of the foot. After molding, the physiatrist reexamined the situation with the participant standing on the insoles to ensure the neutral position of the subtalar joint. The insoles were remolded or modified as necessary. The details of this procedure were described in our previous studies $[9,19,20]$. The treatment group participants each received a pair of highdensity ethyl vinyl acetate thermoplastic foot insoles with medial arch support. Participants were instructed to place the insoles inside comfortable shoes of their choosing. We suggested that shoes with a high, firm heel counter, wide toe box, and flat sole would be the most appropriate selection. The treatment group was instructed to wear the insoles for 1 hour on the first day and to increase the duration of wear by 1 hour per day to reach 5 to 10 hours within 1 week [21]. The control group participants, by contrast, were told they were on a waiting list to receive customized foot insoles after 12 weeks. All children were instructed to wear their shoes as usual at school and for outdoor activities for the duration of the intervention period.

\section{Outcome Measures}

\section{Primary Outcome: Physical Functional Performance}

Physical functional performance was evaluated by walking speed, stairs ascent and descent tests, the Five Times Sit-to-Stand test, and the Timed Up and Go test. Walking speed was calculated with the participant walking at a comfortable speed and at the fastest possible speed across a 10-meter long hard and flat surface. For the stairs ascent and descent tests, participants ascended or descended a flight of stairs (14 steps with a height of $18 \mathrm{~cm}$ each) as fast as possible. The Five Times Sit-to-Stand test evaluated lower limb strength and balance based on how long it took a participant to change from a seated to standing position 5 times. The Timed Up and Go test measured the time needed for a participant to rise from a sitting position, walk 3 meters, turn around, and promptly return to a sitting position. A longer time taken indicated a greater physical functional performance limitation. 


\section{Secondary Outcomes: Physical Functioning and Psychological Well-Being}

The Pediatric Outcome Data Collection Instrument-Parent (PODCI) was used to evaluate physical functioning and psychological well-being. The PODCl consists of 6 domains: upper extremity and physical function, transfer and basic mobility, sports and physical function, pain and comfort, happiness, and global function. Each domain scales from 0 to 100, with 100 representing optimal functioning. The Chinese version of the PODCI has been found to have high reliability [22].

The Child Health Questionnaire-Parent Form (CHQ-PF28) is a parent-reported questionnaire used to assess the physical and psychosocial functioning of children. Our study appraised the participants' wellbeing using 9 of the CHQ-PF28 scales: physical function; role/social functioning: emotional/behavior; role/social functioning: physical, bodily pain, behavior, mental health, self-esteem, general health perceptions, and changes in health [23]. Responses for each scale are summed and transformed into a score of 0 to 100. Higher scores indicate better functioning and quality of life [23].

\section{Statistical Analysis}

For an effect size of 0.86 , an alpha level of 0.05 , and a power of $75 \%$ for a randomized controlled trial with baseline and postintervention observations, at least 40 participants must be included (20 in each group) [24]. Given the probability of a $10 \%$ dropout rate, at least 44 participants (22 participants in each group) should be selected initially. The results are expressed as means and standard deviations. A chisquared test and t-tests were used for baseline characteristics and outcomes comparisons. Paired t-tests were used to examine whether significant improvements occurred in the two groups after intervention, with $T_{1}-T_{0}$ representing the difference between performance after the 12-week intervention $\left(T_{1}\right)$ and performance at baseline $\left(T_{0}\right)$. Student's t-tests were used to compare the improvements between the two groups; $95 \%$ confidence intervals were used. The effect size was estimated, with effect size defined as no effect (0-0.19), small effect (0.20-0.49), intermediate effect (0.50-0.79), and large effect ( 0.80 or higher) [25]. Statistical significance was set at $p<0.05$.

\section{Results}

A total of 45 participants, specifically 25 boys and 20 girls, were analyzed in our study. No adverse event or complication occurred during the intervention period. The mean age of the treatment and control group members was $5.6 \pm 2.5$ and $5.6 \pm 2.4$ years, respectively. Demographic data did not differ between the two groups (Table 1). 
Table 1

Basic Demographics of Participants

\begin{tabular}{|c|c|c|c|}
\hline & Treatment Group & Control Group & \\
\hline Variable & $(n=22)$ & $(n=23)$ & $\mathrm{P}$ \\
\hline \multicolumn{4}{|l|}{ Sex } \\
\hline Male & $10(45.5)$ & $15(65.2)$ & \\
\hline Female & $12(54.5)$ & $8(34.8)$ & \\
\hline Age (year) & $5.6 \pm 2.5$ & $5.6 \pm 2.4$ & .982 \\
\hline $\mathrm{BMI}\left(\mathrm{kg} / \mathrm{m}^{2}\right)$ & $14.8 \pm 1.5$ & $15.1 \pm 1.2$ & .732 \\
\hline Beighton Score & $6.4 \pm 2.3$ & $7.0 \pm 2.1$ & .159 \\
\hline \multicolumn{4}{|l|}{ Navicular drop } \\
\hline Left & $15.9 \pm 4.4$ & $15.0 \pm 3.9$ & .427 \\
\hline Right & $14.1 \pm 3.9$ & $14.0 \pm 4.1$ & .946 \\
\hline Average & $15.0 \pm 3.8$ & $14.5 \pm 3.7$ & .676 \\
\hline \multicolumn{4}{|c|}{ Foot Posture Index } \\
\hline Left & $8.9 \pm 2.7$ & $7.8 \pm 3.1$ & .328 \\
\hline Right & $7.7 \pm 2.4$ & $6.7 \pm 3.4$ & .315 \\
\hline Average & $8.3 \pm 2.5$ & $7.3 \pm 3.1$ & .309 \\
\hline \multicolumn{4}{|c|}{ Abbreviation: BMI, body mass index. } \\
\hline \multicolumn{4}{|c|}{ Values are $n(\%)$, except for age and $B M I$, where values are mean $\pm S D$. } \\
\hline${ }^{\star} P<.05$ & & & \\
\hline
\end{tabular}

Clinical measurements demonstrated that the average Beighton Score was higher than the cutoff value (> 4) in both groups. For the navicular drop test, the measured difference in navicular bone height was 15.0 $\pm 3.8 \mathrm{~mm}$ and $14.5 \pm 3.7 \mathrm{~mm}$ in the treatment group and the control group, respectively. The Foot Posture Index in the treatment group $(8.3 \pm 2.5)$ and the control group $(7.3 \pm 3.1)$ indicated pronated foot for both. No significant difference in physical functional performance, $\mathrm{PODCl}$ scores, or the well-being of childrenaccording to CHQ-PF28 scores-was found at baseline between the groups (Table 2). 
Table 2

Outcome Measures of Participants at Baseline $\left(T_{0}\right)$

\begin{tabular}{|llll|}
\hline Variables & Treatment Group & Control Group & $P$ \\
\hline Physical functional performance & & & \\
\hline Normal speed (second) & $10.6 \pm 3.0$ & $10.7 \pm 1.6$ & .450 \\
\hline Fastest speed & $7.2 \pm 1.4$ & $7.5 \pm 1.2$ & .225 \\
\hline Stairs ascent & $10.2 \pm 3.6$ & $10.0 \pm 2.4$ & .390 \\
\hline Stairs descent & $11.3 \pm 7.2$ & $10.6 \pm 4.6$ & .348 \\
\hline Five Times Sit-to-Stand & $8.7 \pm 2.9$ & $9.3 \pm 3.2$ & .321 \\
\hline Timed Up and Go & $9.3 \pm 2.3$ & $9.2 \pm 1.9$ & .418 \\
\hline PODCl & & & .617 \\
\hline Upper extremity \& physical function & $95.9 \pm 4.8$ & $95.2 \pm 4.7$ & .450 \\
\hline Transfer and basic mobility & $98.9 \pm 2.1$ & $98.2 \pm 3.3$ & .909 \\
\hline Sports \& physical function & $91.1 \pm 8.7$ & $90.7 \pm 9.5$ & .093 \\
\hline Pain/comfort & $88.1 \pm 13.9$ & $81.1 \pm 13.6$ & .244 \\
\hline Happiness & $91.5 \pm 11.2$ & $84.7 \pm 22.8$ & .188 \\
\hline Global function & $93.5 \pm 5.7$ & $91.2 \pm 5.3$ & .105 \\
\hline CHQ-PF28 & & & \\
\hline Well-being of child & $74.6 \pm 20.2$ & $69.1 \pm 14.8$ & \\
\hline $\begin{array}{l}\text { Abbreviation: } T_{0} \text { baseline; PoDCl, Pediatric Outcome Data Collection } \\
\text { Child Health Questionnaire-Parent Form. }\end{array}$ & & & \\
\hline Data presented as mean \pm standard deviation & $($ SD), in seconds. & \\
\hline *P<.05 & & & \\
\hline
\end{tabular}

After the 12-week intervention, the treatment group exhibited significant improvement $\left(T_{1}-T_{0}\right)$ compared with the control group in physical functional performance, including fastest walking speed on level ground (1.3 vs 0.2, $p=0.046$; effect size $=0.515$ ), stairs ascent time ( 2.1 vs $0.07, p=0.023$; effect size $=$ 0.658 ), and stairs descent time ( 2.3 vs $0.07, p=0.012$; effect size $=0.718$; Table 3 ). The changes in $\mathrm{PODCl}$ scores and child well-being, based on the CHQ-PF28, demonstrated no significant difference between the groups after the 12-week intervention (Table 3).

\section{Discussion}


Asymptomatic flexible flat foot is commonly observed in children before the age of 6 [7]. Due to uncertainty regarding the effect of customized MLA foot insoles on healthy children, we recruited children without symptoms in the lower extremities to evaluate the short-term effect of such insoles. We firstly evaluated their physical functional performance, physical and psychosocial function, and well-being based on the ICF framework. Our results demonstrated that wearing foot insoles with MLA support significantly improved children's physical functional performance including walking speed on level ground as well as their stairs ascending/descending times which belong to the domain of activity in the ICF scheme-compared with the control group. Foot insoles did not affect psychosocial status or wellbeing, which belong to the domain of body functions and structures and participation.

In children, the strength of connective tissue and the flexibility of joints reach adult levels by the age of 15 [26]. The muscles and surrounding structures supporting the foot and ankle get stronger and grow denser when children begin to walk. In our previous study, wearing the customized medial arch-support insoles for 12 weeks improved the physical functional performance and activity in children with symptomatic flexible flatfoot [9]. However, Moraleda et al found no difference in hindfoot alignment, longitudinal arch, and the pronation/supination of the forefoot between symptomatic and asymptomatic flexible flatfeet [27]. Relevant studies have outlined how proper footwear enables children to walk with greater ankle and knee motion, increases tibialis anterior activity, and encourages a rearfoot strike pattern [28]. Gait performance can affect the physical functional performance of children, including in the activities of stairs climbing, running, hopping, and balancing. In the present study, we demonstrated that customized arch-support insoles could affect the gait and thus improved the physical functional performance in walking and stairs climbing in healthy children. In addition, a greater variety of commercially manufactured foot insoles with medial arch support, arch height, and hardness are now available. Therefore, comparison between customized and ready-made insoles with MLA support should be investigated in the future.

The participants in our study exhibited the features of flat foot and hypermobility, which have a high prevalence in preschool-age children. In a comparison of custom-made and ready-made foot orthotics for children with flexible pronated feet, the researchers found no significant difference in gross motor proficiency, self-perception, exercise efficiency, or pain between groups [29]. In that study, the navicular drop test produced a similar result to ours, but the average age therein was older than for our participants. The different outcomes may be attributed to 2 reasons. First, the ready-made foot insoles still had a heatmoldable thermoplastic medial arch, which was not the case for our control group. Second, older children have greater lower extremity muscle strength, mature foot arch growth, and stronger connective tissue, which could all reduce the effect of foot insoles. Another cohort study found significant improvement of foot pain and balance in children with symptomatic flexible flat feet after 3 months of use of custommolded foot orthoses [30]. However, they had no control group, and the baseline features, symptoms, and methodology were all different from ours.

Another cross-sectional study involving 155 children aged 8 to 15 reported that those with flexible flat feet exhibited significantly impaired health-related quality of life, especially in the physical domain [31]. In our 
study, the baseline scores of the PODCI were medium to high, meaning that flexible flat feet initially had a relatively small impact on physical function and psychological well-being. However, the ceiling effect might explain why the influence on physical function and the psychological aspect was not significant with the foot insoles used in our study.

\section{Study Limitations}

Some limitations should be noted. First, the parents of participants recruited from the outpatient clinic had a relatively high general awareness of children's health, and because of this selection bias, we cannot generalize our findings to the wider community. Second, subtle symptoms (such as soreness or fatigue over the foot or calf after prolonged walking or running) might not be apparent to parents or the child. Third, we did not divide the participants into subgroups according to the severity of hypermobility or flat feet. The children with borderline or normal structure and function may have diluted the effect of the insoles. Fourth, we evaluated the participants with anthropometric measurements but not radiological examinations such as X-ray, magnetic resonance imaging, or ultrasonography. More subtle structural deformities or pathological conditions may have been overlooked. Finally, we only followed the outcomes for 12 weeks, and the long-term effect was not evaluated.

\section{Conclusions}

This was the first study to evaluate the short-term therapeutic effects of customized arch-support insoles on the physical functional performance, physical function, and psychological well-being of healthy children without symptoms in their lower limbs under the ICF scheme. Our results demonstrated that children using customized arch-support insoles displayed improved physical functional performance which belonged to the domain of activity in the ICF. No significant effect was found on physical function or psychological well-being between the groups at 12-week follow-up. In the future, it may be advisable to use a larger sample size of participants more precisely selected on the basis of the severity of hypermobility or flat feet, with a longer follow-up.

\section{Abbreviations}

CHQ-PF28 (Child Health Questionnaire-Parent Form)

ICF (International Classification of Functioning, Disability and Health)

MLA (Medial longitudinal arch)

PODCI (Pediatric Outcome Data Collection Instrument-Parent)

\section{Declarations}

\section{Contributions}


Ying-Chen Kuo: interpretation of the data and writing the first draft of the manuscript. Ru-Lan Hsieh: study conception and design, material preparation and data collection, interpretation of the data and revising the manuscript. Wen-Chung Lee: study conception and design, material preparation and data collection, statistical analysis. All authors approved the final version of the manuscript as submitted and agree to be accountable for all aspects of the work.

Funding: This study was partially supported by research grants from Shin Kong Wu Ho-Su Memorial Hospital (2018SKHADR028, 2019SKHADR038, 2020SKHADR035, and 2021 SKHADR032) and the Ministry of Science and Technology, Taiwan (MOST-102-2314-B-341-001, MOST 104-2314-B-002-118MY3, MOST-105-2314-B-341-002, MOST-105-2314-B-002-049-MY3, MOST-106-2314-B-341-003-MY2, MOST 108-2314-B-341-003, MOST 108-2314-B-002-127-MY3, and MOST 109-2314-B-341-001). The funders had no role in the study design, data collection, and analysis, decision to publish, or preparation of the manuscript.

Conflicts of interest: The authors certify that there is no conflict of interest with any financial organization regarding the material discussed in the manuscript.

Availability of data and material: Data may be shared upon reasonable requests

Code availability: Not applicable

Ethics approval: The study was reviewed by the Shin Kong Wu Ho-Su Memorial Hospital Institutional Review Board.

Consent to participate: Not applicable

Consent for publication: Not applicable

Acknowledgments: The authors sincerely thank all the participants for their cooperation. The authors thank Yi-Jung Wu for the valuable technical support in this study.

\section{References}

1. Jacobs B (2010) Toe walking, flat feet and bow legs, in-toeing and out-toeing. Paediatrics Child Health 20:221-224

2. Volpon JB (1994) Footprint analysis during the growth period. J Pediatr Orthop 14:83-85. https://doi.org/10.1097/01241398-199401000-00017

3. Stavlas P, Grivas TB, Michas C, Vasiliadis E, Polyzois V (2005) The evolution of foot morphology in children between 6 and 17 years of age: a cross-sectional study based on footprints in a Mediterranean population. J Foot Ankle Surg 44:424-428.

https://doi.org/10.1053/j.jfas.2005.07.023 
4. Walther M, Herold D, Sinderhauf A, Morrison R (2008) Children sport shoes--a systematic review of current literature. Foot Ankle Surg 14:180-189. https://doi.org/10.1016/j.fas.2008.04.001

5. Lin CJ, Lai KA, Kuan TS, Chou YL (2001) Correlating factors and clinical significance of flexible flatfoot in preschool children. J Pediatr Orthop 21:378-382

6. Chang JH, Wang SH, Kuo CL, Shen HC, Hong YW, Lin LC (2010) Prevalence of flexible flatfoot in Taiwanese school-aged children in relation to obesity, gender, and age. Eur J Pediatr 169:447-452. https://doi.org/10.1007/s00431-009-1050-9

7. Pfeiffer M, Kotz R, Ledl T, Hauser G, Sluga M (2006) Prevalence of flat foot in preschool-aged children. Pediatrics 118:634-639. https://doi.org/10.1542/peds.2005-2126

8. Smits-Engelsman B, Klerks M, Kirby A (2011) Beighton score: a valid measure for generalized hypermobility in children. J Pediatr 158:119-123, 123.e1-4. https://doi.org/10.1016/j.jpeds.2010.07.021

9. Hsieh RL, Peng HL, Lee WC (2018) Short-term effects of customized arch support insoles on symptomatic flexible flatfoot in children: A randomized controlled trial. Med (Baltim) 97:e10655. https://doi.org/10.1097/md.0000000000010655

10. Dars S, Uden H, Banwell HA, Kumar S (2018) The effectiveness of non-surgical intervention (Foot Orthoses) for paediatric flexible pes planus: A systematic review: Update. PLoS One 13:e0193060. https://doi.org/10.1371/journal.pone.0193060

11. Lee HJ, Lim KB, Yoo J, Yoon SW, Yun HJ, Jeong TH (2015) Effect of Custom-Molded Foot Orthoses on Foot Pain and Balance in Children With Symptomatic Flexible Flat Feet. Ann Rehabil Med 39:905-913. https://doi.org/10.5535/arm.2015.39.6.905

12. Nakano H, Murata S, Abiko T, Mitsumaru N, Kubo A, Hachiya M, Matsuo D, Kawaguchi M (2020) Effects of Long-Term Use of Insoles with a Toe-Grip Bar on the Balance, Walking, and Running of Preschool Children: A Randomized Controlled Trial. Biomed Res Int 2020:1940954. https://doi.org/10.1155/2020/1940954

13. Ustün TB, Chatterji S, Bickenbach J, Kostanjsek N, Schneider M (2003) The International Classification of Functioning, Disability and Health: a new tool for understanding disability and health. Disabil Rehabil 25:565-571. https://doi.org/10.1080/0963828031000137063

14. Remvig L, Jensen DV, Ward RC (2007) Are diagnostic criteria for general joint hypermobility and benign joint hypermobility syndrome based on reproducible and valid tests? A review of the literature. J Rheumatol 34:798-803

15. Adhikari U, Arulsingh W, Pai G et al (2014) Normative values of navicular drop test and the effect of demographic parameters - a cross sectional study. Ann Biol Res 5:40-48

16. Deng J, Joseph R, Wong CK (2010) Reliability and validity of the sit-to-stand navicular drop test: Do static measures of navicular height relate to the dynamic navicular motion during gait. J Stud Phys Ther Res 2:21-28

17. Evans AM, Nicholson H, Zakarias $N$ (2009) The paediatric flat foot proforma (p-FFP): improved and abridged following a reproducibility study. J Foot Ankle Res 2:25. https://doi.org/10.1186/1757- 


\section{$1146-2-25$}

18. Levinger P, Menz HB, Fotoohabadi MR et al (2010) Foot posture in people with medial compartment knee osteoarthritis. J Foot Ankle Res 3:29. https://doi.org/10.1186/1757-1146-3-29

19. Hsieh RL, Lee WC (2014) Immediate and medium-term effects of custommoulded insoles on pain, physical function, physical activity, and balance control in patients with knee osteoarthritis. J Rehabil Med 46:159-165. https://doi.org/10.2340/16501977-1254

20. Hsieh RL, Lee WC (2016) Clinical effects of lateral wedge arch support insoles in knee osteoarthritis: a prospective double-blind randomized study. Med (Baltim) 95:e3952. https://doi.org/10.1097/md.0000000000003952

21. Bennell KL, Bowles KA, Payne $C$ et al (2011) Lateral wedge insoles for medial knee osteoarthritis: 12 month randomised controlled trial. BMJ 342:d2912. https://doi.org/10.1136/bmj.d2912

22. Hsieh RL, Lin MI, Huang HY et al (2011) The relationship between the Pediatric Outcomes Data Collection Instrument and functional impairment in developmentally delayed Chinese children and their parents' health: implications for child and family-centered medicine. Int J Pers Cent Med 1:1-8

23. Marklund B, Ahlstedt S, Nordstrom G (2006) Health-related quality of life in food hypersensitive schoolchildren and their families: parents' perceptions. Health Qual Life Outcomes 4:48. https://doi.org/10.1186/1477-7525-4-48

24. Noordzij M, Tripepi G, Dekker FW, Zoccali C, Tanck MW, Jager KJ (2010) Sample size calculations: basic principles and common pitfalls. Nephrol Dial Transplant 25:1388-1393. https://doi.org/10.1093/ndt/gfp732

25. Cohen J (1988) Statistical Power Analysis for the Behavioral Sciences, 2nd edn. Erlbaum Associates, Hillsdale

26. Walther M, Herold D, Sinderhauf A, Morrison R (2008) Children sport shoes--a systematic review of current literature. Foot Ankle Surg 14:180-189. https://doi.org/10.1016/j.fas.2008.04.001

27. Moraleda L, Mubarak SJ (2011) Flexible flatfoot: differences in the relative alignment of each segment of the foot between symptomatic and asymptomatic patients. J Pediatr Orthop 31:421428. https://doi.org/10.1097/bpo.0b013e31821723ce

28. Wegener C, Hunt AE, Vanwanseele B, Burns J, Smith RM (2011) Effect of children's shoes on gait: a systematic review and meta-analysis. J Foot Ankle Res 4:3. https://doi.org/10.1186/1757-1146-4-3

29. Whitford D, Esterman A (2007) A randomized controlled trial of two types of in-shoe orthoses in children with flexible excess pronation of the feet. Foot Ankle Int 28:715-723. https://doi.org/10.3113/fai.2007.0715

30. Lee HJ, Lim KB, Yoo J, Yoon SW, Yun HJ, Jeong TH (2015) Effect of Custom-Molded Foot Orthoses on Foot Pain and Balance in Children With Symptomatic Flexible Flat Feet. Ann Rehabil Med 39:905-913. https://doi.org/10.5535/arm.2015.39.6.905

31. Kothari A, Stebbins J, Zavatsky AB, Theologis T (2014) Health-related quality of life in children with flexible flatfeet: a cross-sectional study. J Child Orthop 8:489-496. https://doi.org/10.1007/s11832014-0621-0 


\section{Tables}

Due to technical limitations, Table 3 is only available as a download in the Supplemental Files section.

\section{Figures}

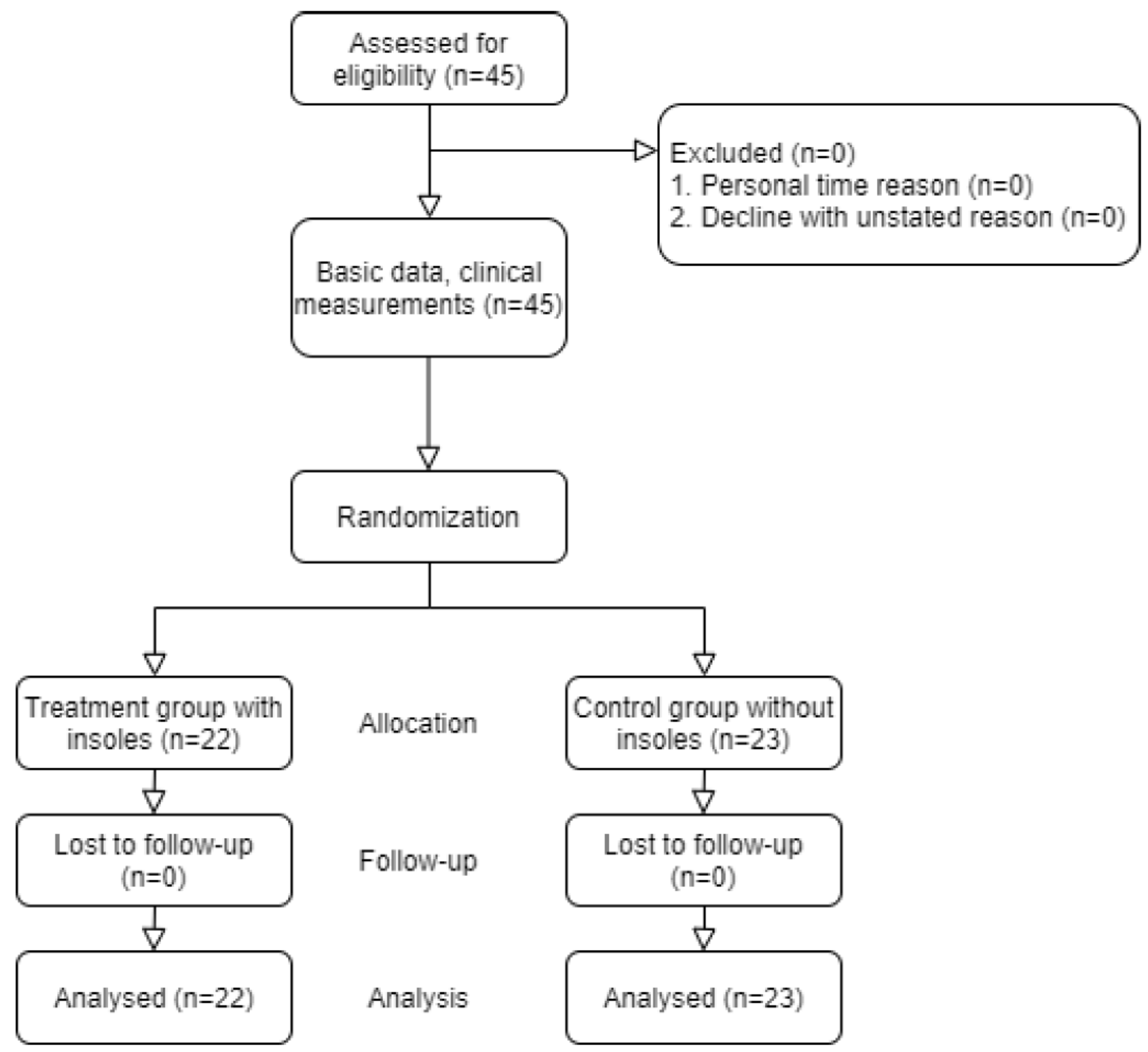

Figure 1

Flow Diagram of Participant Enrollment

\section{Supplementary Files}


This is a list of supplementary files associated with this preprint. Click to download.

- Table3.docx

- CONSORTEU.doc 